\title{
Desarrollo infantil temprano y localización auditiva en niños ciegos: una revisión*
}

\author{
Early Infant Development and Auditory \\ Localization in Blind Children: A Review
}

Recibido: junio 13 de 2012 | Revisado: diciembre 17 de 2012 | Aceptado: marzo 28 de 2013

\author{
MERCEDES XIMENA HÜG ** \\ Claudia ARIAS *** \\ Universidad Nacional de Córdoba, Argentina
}

doi:10.11144/Javeriana.UPSY13-1.dilt

Para citar este artículo: Hüg, M. X. \& Arias, C. (2014). Desarrollo infantil temprano y localización auditiva en niños ciegos: una revisión. Universitas Psychologica, 13(1), 71-82. doi:10.11144/Javeriana. UPSY13-1.dilt

* Artículo de revisión teórica. Esta revisión fue posible gracias a becas de formación de postgrado recibidas por Mercedes X. Hüg, primero de la Secretaría de Ciencia y Tecnología (SECyT) de la Universidad Nacional de Córdoba (UNC) y posteriormente del Consejo Nacional de Investigaciones Científicas y Técnicas (CONICET) y al subsidio "Localización de sonidos directos y reflejados y detección de la altura tonal de la repetición en infantes con visión normal y ciegos", dirigido por Claudia Arias y codirigido por Oscar Ramos (PID SECyT. Universidad Nacional de Córdoba. Res. Rect. 2254/06).

*** Centro de Investigación y Transferencia en Acústica (CINTRA), Universidad Tecnológica Nacional, Facultad Regional Córdoba, Argentina. Facultad de Psicología, Universidad Nacional de Córdoba, Córdoba, Argentina.

*** Consejo Nacional de Investigaciones Científicas y Técnicas (CONICET).

\section{RES UMEN}

El estudio científico de la habilidad para localizar sonidos en niños con visión normal se inició a comienzos de los 80 . Sin embargo, en el caso de los niños ciegos, las investigaciones realizadas sobre este tema son exiguas. En el presente trabajo se presenta una revisión de artículos sobre el desarrollo motor y cognitivo de los niños ciegos que evaluaron conductas de localización sonora en la primera infancia. En primer lugar, se sintetizan trabajos pioneros realizados en la década del 60 y luego se presentan investigaciones realizadas a partir de la década del 80 . Por último, se analizan e integran los principales resultados de estos trabajos, se discuten sus implicancias y se plantean algunos de los interrogantes aún no resueltos en este campo del conocimiento,

\section{Palabras clave autores}

Localización auditiva, desarrollo infantil, ceguera.

Palabras clave descriptores

Psicología del desarrollo, revisión, cognición, control motor.

\section{A B S T R A C T}

The scientific study of the ability to localize sounds in children with normal vision began in the early 80 s. However, in the case of blind children, scarce research has been conducted on this subject. This paper presents a review of articles on cognitive and motor development of blind children that evaluated sound localization behaviors in early childhood. First, pioneering studies published in the 60s are summarized and then researches from the $80 \mathrm{~s}$ are presented. Finally, the main findings of these papers are analyzed and integrated, their implications are discussed and some questions unresolved in this field of knowledge are stated.

Keywords authors

Auditory localization, child development, blindness.

Keywords plus

Development psychology, review, cognition, motor control. 


\section{Introducción}

La audición permite orientar y dirigir la atención hacia los eventos sonoros relevantes del ambiente. Mientras que la percepción visual se restringe a una ventana frontal, el sistema auditivo es capaz de detectar y monitorear las posiciones de eventos sonoros en el espacio que se extiende alrededor de la cabeza en todas las direcciones, permitiendo que la persona se oriente y procese visualmente los estímulos relevantes (Grantham, 1995; Perrott, Saberi, Brown \& Strybel, 1990).

La audición espacial es el campo temático que estudia los procesos involucrados en la habilidad para determinar de dónde proviene el sonido, a qué distancia y en qué dirección se encuentra la fuente sonora ${ }^{1}$ que lo origina, de qué evento sonoro se trata y cuáles son sus características. La localización auditiva hace referencia a la percepción de la posición de una fuente sonora en el plano horizontal (izquierda-derecha), vertical (arriba-abajo) y a la percepción de la distancia relativa entre participante y fuente (Blauert, 1997). En el hombre, la habilidad para localizar sonidos es muy buena en el plano horizontal, algo menos eficiente en el plano vertical y la precisión con la que se percibe la distancia es regular (Moore, 1995).

La gran mayoría de las investigaciones sobre localización auditiva han sido realizadas con participantes adultos. Aunque en la segunda mitad del siglo XX se produjo un considerable avance en la comprensión de las competencias perceptuales de los bebés, la investigación se refirió en gran medida a la percepción visual (Clifton, 2001). Los estudios experimentales sobre el desarrollo de la audición espacial en niños pequeños comenzaron recién a comienzos de la década del 80 (Hüg \& Arias, 2009). La adaptación de procedimientos psicofísicos clásicos para su aplicación en bebés, permitió demostrar que la conducta de los niños varía de una manera predecible según ciertos parámetros de la

1 Se denomina fuente sonora a cualquier cuerpo que vibra en contacto con un medio elástico (sólido, líquido o gaseoso) y transmite mecánicamente estos movimientos produciendo sonido (American National Standard Institute [ANSI], 1999). estimulación sonora (Schneider \& Trehub, 1992). Algunos de los interrogantes que se plantearon los investigadores fueron: icuándo comienzan los bebés a localizar fuentes sonoras?, icómo se modifica el rendimiento con la edad?, ia qué edad comienzan a percibir la distancia a la que se encuentra un objeto sonoro?

Fueron Muir y Field en el año 1979 los primeros en demostrar que bajo ciertas condiciones experimentales, a pocos minutos de nacer, los bebés giran su cabeza en la dirección de una fuente sonora

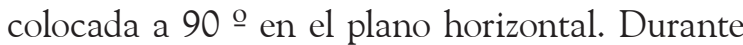
los primeros meses de vida, se observan notables cambios en la respuesta de orientación de la cabeza: en los recién nacidos es lenta y poco precisa, disminuye como respuesta motora confiable y válida entre el primer y segundo mes de vida, reaparece hacia los 3 o 4 meses de edad. A los 4 o 5 meses este comportamiento es cualitativamente muy diferente de la respuesta del neonato: la latencia en iniciar el movimiento disminuye de 7 segundos a 2 o 3 segundos, es más precisa y está acompañada de búsqueda visual (Field, Muir, Pilon, Sinclair \& Dodwell, 1980; Muir, Clifton \& Clarkson, 1989). A los 4 meses de edad, los niños logran alcanzar con éxito un juguete sonoro presentado por el experimentador en una sala a oscuras, sin claves visuales ni táctiles previas sobre su posición (Clifton, Muir, Ashmead \& Clarkson, 1993; Hillier, Hewitt \& Morrongiello, 1992). A los 6 meses, investigaciones aún muy incipientes indican que los bebés poseen la capacidad de discriminar, sin utilizar claves visuales, si un objeto sonoro se encuentra o no suficientemente cerca de su cuerpo como para alcanzarlo (Clifton, Perris \& Bullinger, 1991; Hüg, 2011).

Entre los 6 y 18 meses, la evidencia empírica obtenida ha permitido determinar que la habilidad de los niños para percibir pequeños cambios en la posición de fuentes sonoras mejora significativamente con la edad (Hüg, 2011; Morrongiello, 1987, 1988; Morrongiello \& Rocca, 1987, 1990). A los 5 años y bajo ciertas condiciones experimentales, los niños obtienen umbrales similares a los de los adultos (Litovsky, 1997). Esta mejora en el rendimiento de los niños durante los primeros años de vida se ha explicado por la maduración del sistema auditivo 
y otros factores estrechamente interrelacionados entre sí, vinculados al desarrollo motor y cognitivo (Hüg \& Arias, 2009).

En relación con los niños ciegos, la bibliografía científica existente sobre las características de su desarrollo es exigua y contradictoria. Esto se explica, en parte, por la dificultad para conformar muestras homogéneas de niños ciegos sin patologías agregadas y que cuenten con diagnósticos precisos sobre su patología visual. Al respecto, cabe señalar que se ha estimado que la prevalencia de patologías asociadas a la ceguera alcanza al 70\% de la población de personas ciegas (Mervis, Boyle $\&$ Yeargin-Allsopp, 2002). Específicamente, varios autores llaman la atención acerca de la escasez de investigaciones que indaguen sobre el desarrollo de la audición espacial en niños ciegos (Humphrey, Dodwell, Muir \& Humphrey, 1988; Hüg \& Arias, 2006; Warren, 1984). En general, se evalúan conductas de localización auditiva como girar la cabeza hacia una fuente sonora o alcanzar un objeto sonoro, en el marco de investigaciones sobre el desarrollo motor y cognitivo del niño. Los estudios realizados generalmente son descriptivos, muy pocos emplean diseños experimentales y revelan grandes diferencias individuales.

En el presente trabajo, se presenta una revisión de artículos sobre el desarrollo motor y cognitivo de los niños ciegos, que evaluaron conductas de localización sonora en la primera infancia. En primer lugar, se sintetizan trabajos pioneros realizados en la década del 60 y luego se presentan investigaciones realizadas a partir de la década del 80 . Por último, se analizan e integran los principales resultados de estos trabajos, se discuten sus implicancias y se plantean algunos de los interrogantes aún no resueltos en este campo del conocimiento.

\section{Estudios de la década del 60}

Las primeras observaciones sistemáticas sobre el desarrollo de conductas de localización auditiva en niños ciegos en la temprana infancia se efectuaron en la década del 60. Consistían, en general, en estudios de caso sobre el desarrollo motor y cognitivo realizados por profesionales especialistas en esti- mulación temprana que registraban las conductas espontáneas que estos niños realizaban en sus hogares o el consultorio. En 1964, Fraiberg y Freedman publicaron observaciones sobre dos niños ciegos: una niña que fue evaluada desde los 5 hasta los 28 meses y un niño de 9 años. En el caso de la niña ciega, se observó que desde los 5 y hasta aproximadamente los 7 meses de edad, su desarrollo motor grueso siguió el curso evolutivo esperado para la edad: por ejemplo, logró el sostén cefálico y se sentó sin ayuda a edades similares a las de los bebés con visión normal. Sin embargo, logró alcanzar objetos sonoros recién a los 10 meses, mientras que, como se mencionó, en los niños con visión normal esta conducta se observa alrededor de los 4 meses de edad. En el mismo artículo, los autores compararon este caso con el del niño ciego de 9 años, que sin poseer otras discapacidades asociadas, había alcanzado la marcha autónoma recién a los 4 años. Durante su temprana infancia, este niño había mostrado muy poco interés en utilizar las manos para manipular objetos, en general no había intentado recuperar objetos sonoros cuando se le caían y permanecía durante períodos prolongados de tiempo acostado en la cama o en el suelo sin interés por explorar el entorno.

En el mismo año, Burlingham (1964) publicó observaciones sobre el desarrollo de conductas de orientación auditiva en un bebé ciego desde los 6 meses a los 19 meses. Señaló que a los 6 meses el bebé sonreía cuando la madre hacía sonar un sonajero, realizaba un leve giro de cabeza cuando el reloj daba la hora, detenía su actividad espontánea cuando corrían las cortinas de la habitación. A los 7 meses disfrutaba produciendo sonido: estrujaba papel, rasguñaba almohadones. A los 19 meses extendía la mano para buscar a la persona que hablaba y se desanimaba si no podía lograrlo.

En 1966, Fraiberg, Siegel y Gibson publicaron observaciones sobre el desarrollo de un niño con ceguera congénita desde los 5 hasta los 20 meses de edad. A los 6 meses, el niño tenía un desarrollo motor acorde a la edad, se mostraba atento a voces y sonidos de la casa. Sin embargo, si se le retiraba un objeto sonoro de las manos no evidenciaba ningún interés en recuperarlo. Los 
primeros intentos de alcanzar un juguete sonoro se observaron entre los 7 y 9 meses y, a esta edad, generalmente no eran exitosos. Entre los 9 y 11 meses, si se le quitaba de las manos un objeto sonoro y se lo colocaba en una nueva posición, el niño lo buscaba teniendo en cuenta principalmente las primeras claves táctiles de localización y solo en algunas ocasiones era capaz de utilizar las claves sonoras que indicaban la nueva posición del objeto. Aproximadamente a los 12 meses, intentó por primera vez alcanzar objetos basándose solo en claves sonoras. Los autores señalan que dos días después de este logro, comenzó a desplazarse arrastrándose para buscar objetos sonoros que estaban en el suelo más allá de su alcance. A los 14 meses, logró seguir con el giro de la cabeza, la trayectoria de un juguete movido por el experimentador a la altura de su rostro y asirlo en base a claves sonoras. Postularon que los bebés ciegos se demoran respecto a los niños con visión normal en intentar alcanzar objetos sonoros debido a la complejidad cognitiva de esta tarea: si no se les provee de claves táctiles previas, no logran darse cuenta de que el sonido proviene de un objeto que está "ahí afuera", debido a que aún no han adquirido la noción de permanencia del objeto. Según los autores, en ausencia de claves visuales o táctiles "el sonido solo no confiere sustancialidad al objeto" (p. 329).

Posteriormente, Fraiberg (1968) publicó un estudio longitudinal de 10 años de duración, con 10 niños con ceguera sin otras patologías asociadas de entre 1 y 8 meses de edad corregida. Los resultados mostraron que los niños ciegos lograron alcanzar un juguete sonoro a la edad mediana de 8.5 meses de edad corregida, con un rango de 6 a 11 meses. Además, se observaron importantes demoras en el logro de conductas de locomoción autoiniciadas como arrastrarse, gatear y caminar sin ayuda. Esta última observación coincide con lo reportado en un estudio realizado por Norris, Spaulding y Brodie en 1957, en el que se informó que más del $50 \%$ de los niños ciegos congénitos que se evaluaron, caminaron en forma independiente recién a los 2 o 3 años en promedio, con un rango de 15 meses a 5 años.

\section{Estudios de la década del 80 a la actualidad}

Basándose en el trabajo de Fraiberg et al. (1966), Bigelow (1986) evaluó el rol de las claves sonoras y táctiles en el desarrollo de la conducta de alcanzar objetos mediante un estudio longitudinal en el que participaron 5 niños ciegos congénitos con ceguera total o visión luz, con edades al iniciar el estudio comprendidas entre los 11 y 32 meses. La experiencia se realizó en la casa de cada uno de los niños, a los que visitó en forma mensual. La tarea consistía en alcanzar un juguete sonoro o silente que era presentado por el experimentador a una distancia cercana. En la Tabla 1 se muestra para cada niño evaluado, la edad en meses en la que resolvió con éxito cada una de las condiciones de presentación del juguete.

Sobre la base de estos resultados, la autora postuló la existencia de una secuencia de dificultad creciente de las tareas 1 a 11 en el desarrollo de la conducta de alcanzar objetos. Sin embargo, señala que el tamaño reducido de la muestra y las diferentes edades a las que se comenzó a evaluar a cada niño dificultan la generalización de los resultados. Al igual que Fraiberg et al. (1966), Bigelow (1986) observó que en los niños más pequeños, las claves táctiles fueron más salientes que las sonoras para elicitar y guiar la respuesta de alcanzar el juguete sonoro: en las pruebas en las que el examinador retiraba el juguete, los bebés tendían a dirigir su mano a la posición en la que habían tocado el objeto, ignorando en algunos casos la posición que indicaban las claves sonoras. La autora concluyó que en edades tempranas, la búsqueda de objetos sonoros se limita a la línea media. Posteriormente, logran localizar sonidos en otras posiciones del plano horizontal y en el plano vertical. En una publicación posterior, Bigelow (1992) relacionó los resultados obtenidos por los niños ciegos a, b y c evaluados en esta prueba con datos relativos a su desarrollo motor grueso. Este análisis permitió determinar que estos niños comenzaron a caminar sin ayuda luego de haber logrado completar exitosamente las tareas 8,9 o 10 , lo cual indicaría, según la autora, que la emergencia de habilidades de locomoción y el conocimiento que los niños poseen sobre las propiedades de los objetos están relacionados. 
Posteriormente, Leonhardt (2000) aplicó la escala de Brazelton (a excepción de los ítems que evaluaban conductas con componentes visuales), a 8 bebés con ceguera total y baja visión de entre 8 días y un mes de edad. Para evaluar conductas de habituación al sonido y de localización auditiva de fuentes sonoras ubicadas a la izquierda o derecha, utilizó el sonido de un sonajero y la propia voz del examinador. Los resultados mostraron que las respuestas motoras generales y de alerta de los niños con discapacidad visual fueron similares a las esperadas para neonatos con visión normal. Sin embargo, el tono muscular y las respuestas de reacción cuando se los llevó de la posición acostado a la de sentado, fueron más bajas en los niños ciegos. Las respuestas de alerta al sonido se habituaron más lentamente en los bebés ciegos y con baja visión respecto a lo reportado para bebés con visión normal. En relación con la localización auditiva, se observaron respuestas diferenciadas en función del tipo de estímulo. Cuando se les presentaba la voz del examinador, los bebés giraban lentamente

TABLA 1

Edad en meses en la que los niños ciegos resolvieron exitosamente las condiciones del estudio de Bigelow (1986)

\begin{tabular}{|c|c|c|c|c|c|c|}
\hline \multirow{2}{*}{\multicolumn{2}{|c|}{ Condiciones de presentación del juguete }} & \multicolumn{5}{|c|}{ Participantes* } \\
\hline & & \multirow{2}{*}{20} & \multirow{2}{*}{ b (13) } & \multirow{2}{*}{$\frac{c(32)}{32}$} & \multirow{2}{*}{$\mathrm{d}(11)$} & \multirow{2}{*}{$\frac{\mathrm{e}(15)}{15}$} \\
\hline $1 \mathrm{a}$ & $\begin{array}{l}\text { Juguete silente en posición fija en contacto con el cuerpo } \\
\text { del niño (extremidades o tronco) }\end{array}$ & & & & & \\
\hline $1 b$ & $\begin{array}{l}\text { Juguete sonoro en posición fija en contacto con el } \\
\text { cuerpo del niño (extremidades o tronco) }\end{array}$ & 23 & 13 & 32 & 12 & 20 \\
\hline 2 & $\begin{array}{l}\text { Juguete sonoro en posición fija enfrente del niño con } \\
\text { claves táctiles previas }^{2}\end{array}$ & 23 & 13 & 32 & 14 & - \\
\hline $3 a$ & $\begin{array}{l}\text { Juguete sonoro en posición fija enfrente del niño sin } \\
\text { claves táctiles previas }\end{array}$ & 23 & 13 & 32 & 15 & - \\
\hline $3 b$ & $\begin{array}{l}\text { Juguete sonoro en posición fija izquierda o derecha sin } \\
\text { claves táctiles previas }\end{array}$ & 23 & 13 & 32 & - & - \\
\hline 4 & $\begin{array}{l}\text { Juguete sonoro en movimiento de } 180^{\circ} \text { a la altura del } \\
\text { rostro del niño sin claves táctiles previas }\end{array}$ & 23 & 13 & 32 & - & - \\
\hline $5 a$ & $\begin{array}{l}\text { Búsqueda del juguete silente caído con claves táctiles } \\
\text { previas }\end{array}$ & 24 & 13 & \# & 14 & - \\
\hline $5 b$ & $\begin{array}{l}\text { Búsqueda del juguete sonoro caído con claves táctiles } \\
\text { previas }\end{array}$ & 25 & $\#$ & 32 & 15 & - \\
\hline 6 & $\begin{array}{l}\text { Búsqueda del objeto sonoro escondido bajo una tela sin } \\
\text { claves táctiles previas }\end{array}$ & 26 & 16 & 32 & - & - \\
\hline 7 & $\begin{array}{l}\text { Juguete sonoro en posición fija en el plano vertical } \\
\text { (arriba o abajo) sin claves táctiles previas }\end{array}$ & 25 & 17 & 32 & - & - \\
\hline 8 & $\begin{array}{l}\text { Juguete silente en posición fija enfrente del niño con } \\
\text { claves táctiles previas }\end{array}$ & 28 & 17 & 35 & - & - \\
\hline 9 & $\begin{array}{l}\text { Juguete sonoro, primero en movimiento y luego en } \\
\text { posición fija a } 90^{\circ} \text { con claves táctiles previas }\end{array}$ & 30 & 21 & 34 & - & - \\
\hline 10 & $\begin{array}{l}\text { Juguete con sonido intermitente en movimiento } 180^{\text {O }} \\
\text { sin claves táctiles previas }\end{array}$ & 32 & 22 & 35 & - & - \\
\hline 11 & $\begin{array}{l}\text { Juguete con sonido intermitente en posición fija sin } \\
\text { claves táctiles previas }\end{array}$ & - & - & - & - & - \\
\hline uent & $\begin{array}{l}\text { paréntesis se consigna la edad en meses en la que el niño fue } \\
\text { n indica que el niño no logró la conducta mientras duró el est } \\
\text { meral señala que no pudo completarse la evaluación del niño } \\
\text { Bigelow (1986). }\end{array}$ & $\begin{array}{l}\text { uado } \mathrm{p} \\
\text { sa pru }\end{array}$ & lera & & & \\
\hline
\end{tabular}

2 El procedimiento implementado para brindar claves táctiles previas consistía en colocar el juguete en la mano del niño para que juegue con él, retirarlo después de un momento y colocarlo luego en diferentes posiciones según lo que correspondiera en cada condición.

3 En las condiciones 10 y 11 se observó si el niño intentaba alcanzar el juguete en el momento en el que el objeto no producía sonido. 
la cabeza hasta enfrentar con el rostro a la persona que hablaba. Cuando se les presentaba el sonajero, los bebés permanecían quietos con un alto grado de alerta y expectación frente al sonido, sin mover la cabeza. La autora propone que las demoras observadas pueden deberse, al menos en parte, a que en los primeros meses de vida estos niños suelen permanecer quietos durante períodos prolongados de tiempo, para captar más eficientemente los estímulos sonoros.

En un trabajo ulterior, Leonhardt (2001) señaló que la conducta de giro de cabeza para enfrentar con el rostro una fuente sonora desaparece habitualmente en los niños ciegos luego del primer mes de vida. Luego de un corto período, 15 días en promedio, vuelve a aparecer pero con adaptaciones funcionales: algunos bebés giran su cabeza de manera tal que es el oído el que enfrenta la fuente, como si "miraran con el oído". Postuló que en el segundo mes de vida se ponen en marcha "mecanismos adaptativos que le permiten una mejor orientación de su oído para recoger la mayor cantidad de información posible" (Leonhardt, 2001, p. 211) y que luego de algunos meses amplían su repertorio de conducta, pudiendo girar la cabeza de manera a enfrentar la fuente con su oreja o su rostro, o también permanecer en una postura intermedia con la cabeza inclinada sobre el pecho. La autora menciona que, en general, en los niños ciegos se observa una preferencia por responder enfrentando la fuente con un mismo oído.

Por su parte, Fazzi et al. (2002) implementaron un estudio longitudinal en el que evaluaron la conducta de alcanzar objetos sonoros ubicados cerca del niño (20 a $30 \mathrm{~cm}$ de su cuerpo) y a mayor distancia (más de $30 \mathrm{~cm}$ de su cuerpo) en 20 niños ciegos sin y con problemas neurológicos asociados (hidrocefalia e hipoplasia de cuerpo calloso, entre otros). La edad media de los participantes al comenzar el estudio fue de 11 meses con un rango de 4 a 30 meses. Los resultados mostraron que los niños ciegos sin patología asociada comenzaron a alcanzar objetos sonoros ubicados cerca y a mayor distancia, a los 12 meses en promedio (rango de 9 a 16 meses) y a los 14 meses en promedio (rango de
10 a 18 meses), respectivamente. Los niños con patología neurológica asociada lo hicieron a una edad más avanzada, a los 24 meses en promedio (rango 13 a 33 meses) y a los 19 meses en promedio (rango 15 a 24 meses), respectivamente.

Recientemente, Fazzi et al. (2011) implementaron un estudio longitudinal en el que aplicaron periódicamente el protocolo elaborado por Bigelow (1986) en otra muestra de 37 niños ciegos sin y con problemas neurológicos asociados (síndrome de Joubert, parálisis cerebral, entre otros). La edad media de los niños del primer grupo fue de 9 meses, con edades al iniciar el estudio comprendidas entre los 4 y 24 meses; la edad media del segundo grupo fue de 11 meses, con edades iniciales de entre 6 y 28 meses. Los resultados mostraron que a los 36 meses, la mayoría de los niños sin problemas neurológicos había logrado completar la totalidad del protocolo. Aproximadamente a los 12 meses, este grupo había resuelto correctamente las tareas 1 a 5. En la misma dirección que el artículo de referencia, para los niños fueron más fáciles las tareas en las que se les proveía de claves táctiles y auditivas que aquellas en las que solo contaban con claves auditivas. Las tareas 6 y 8 fueron más difíciles que las anteriores y en las tareas 7 a 11 se observaron las mayores diferencias individuales. El grupo de niños ciegos con problemas neurológicos tuvo un rendimiento significativamente más bajo, los autores señalan que cuando se les presentaba el objeto sonoro estos niños permanecían en general quietos o se evidenciaban estereotipias de hábito motor.

Finalmente y debido a su relevancia, se incluye también en la presente revisión un estudio realizado por Ashmead et al. (1998) con niños y jóvenes con ceguera congénita o adquirida de entre 6 y 20 años (edad media de 14 años) y participantes con visión normal de entre 12 y 15 años (edad media de 13 años). Los autores compararon el rendimiento de estos grupos en tres pruebas de audición espacial: 1) discriminación de cambios en la posición de la fuente sonora en el plano horizontal, vertical y de distancia; 2) identificación de la posición de objeto sonoro en el plano horizontal, vertical y en distancia y 3) locomoción hasta una 
fuente sonora. En la prueba 1) se le presentaron al participante a) sonidos en el plano horizontal que al principio provenían del frente $(0 \stackrel{\circ}{)}$ y luego cambiaban hacia la izquierda o la derecha, b) sonidos en el plano horizontal desde una posición de referencia de $45^{\circ}$ y que luego se desplazaban hacia la izquierda o la derecha de esta posición, c) sonidos en el plano vertical que al principio provenían del frente a la altura del rostro del participante y luego se desplazaban hacia arriba o hacia abajo, d) sonidos desde una distancia inicial de $175 \mathrm{~cm}$ que luego se movían hacia delante o hacia atrás. La tarea de los participantes consistía en reportar verbalmente si habían percibido un cambio en la posición (pruebas a, b, o c) o en la distancia (prueba d) de los sonidos.

En la prueba 2) se utilizó la técnica de alcanzar un objeto sonoro. Para ello el experimentador se ubicó enfrente del participante y le presentó sonidos provenientes de un pequeño parlante colocado en 12 regiones en el plano horizontal (izquierda, derecha o enfrente), vertical (arriba a la altura de la frente o abajo a la altura del torso) y a distintas distancias (cerca, lejos). La tarea de los participantes consistía en señalar con la mano la posición en la que percibían la fuente sonora. En la prueba 3) los autores presentaron sonidos provenientes de un parlante ubicado a 3, 4, 5, 6 ó 7 metros. La tarea de los participantes consistía en caminar hasta la fuente bajo dos condiciones experimentales: estacionaria y dinámica. En la condición estacionaria el sonido cesaba y recién entonces el participante caminaba hasta la fuente. En la condición dinámica, el parlante continuaba emitiendo sonidos mientras el participante caminaba hacia la fuente. Los resultados obtenidos indicaron que el rendimiento de los niños y jóvenes ciegos fue similar y, en algunos casos, como cuando debían discriminar distancia, superior al de los participantes con visión normal. Sin embargo, señalan que debido a que fueron evaluados muy pocos niños menores de 8 años, los resultados no pueden generalizarse a la población de niños ciegos en la primera infancia. Los autores concluyen que el desarrollo de la audición espacial no dependería de la experiencia visual tempra- na y que, en ausencia de visión, posiblemente el sistema auditivo se calibra o ajusta a partir de la percepción de los cambios en las claves auditivas que causa el propio movimiento del niño, como girar la cabeza o caminar.

\section{Discusión}

La mayoría de lo que se conoce en humanos sobre la localización auditiva proviene de investigaciones realizadas con participantes adultos (Hüg \& Arias, 2009). Considerablemente más escasos son los antecedentes que analizan el desarrollo de esta habilidad en niños ciegos en la temprana infancia. En general, se evalúan conductas de localización auditiva en el marco de investigaciones más amplias sobre el desarrollo motor y cognitivo del niño. Los estudios realizados con niños ciegos en la primera infancia son descriptivos, incluyen muestras reducidas de participantes; no aplican procedimientos psicofísicos para estimar, por ejemplo, umbrales localización; ni reportan información sobre los parámetros acústicos de los estímulos sonoros empleados, que en su mayoría consisten en el sonido producido por juguetes sonoros y la voz materna o del experimentador. En cuanto a las variables de respuesta infantil, las más observadas han sido el giro de la cabeza, el movimiento de brazos y manos, y cambios en el estado global del niño, por ejemplo, el aquietamiento (Warren, 1984).

Los estudios realizados indican que, poco después de nacer, las respuestas de localización auditiva de los bebés ciegos siguen un patrón similar a la de los niños con visión normal: se muestran atentos y alertas ante la presencia de sonidos y giran su cabeza en la dirección de fuentes sonoras ubicadas a la izquierda o derecha (Leonhardt, 2000). En ambos grupos se observan también cambios en la respuesta de orientación de la cabeza durante los primeros meses de vida: el giro de cabeza desaparece como respuesta motora confiable y válida entre el primer y segundo mes de vida y vuelve a aparecer aproximadamente a los 3 meses de edad. En el caso de los niños con visión normal, a esta edad la respuesta es más precisa y está acompañada 
de búsqueda visual (Field et al., 1980; Muir et al., 1989). En el caso de los niños ciegos, se describen adaptaciones funcionales para optimizar la recepción de la información auditiva: algunos bebés giran su cabeza de manera tal que es el oído el que enfrenta la fuente, como si "miraran con el oído" (Leonhardt, 2001).

También en etapas tempranas se evidencia que, al igual que para los niños con visión normal, a los niños ciegos resulta más fácil localizar sonidos en el plano horizontal que en el vertical (Bigelow, 1986). Sin embargo, mientras que los niños con visión normal logran alcanzar con éxito un juguete sonoro presentado por el experimentador sin claves visuales ni táctiles previas, a los 4 meses de edad (Clifton et al., 1993; Hillier et al., 1992), se describen grandes diferencias individuales en el logro de esta conducta en los niños ciegos, en algunos casos se observa a los 9 meses y en otros recién después de los 20 meses de edad. Como se mencionó anteriormente, la ausencia de estudios que midan umbrales de localización en niños ciegos menores a 6 años o brinden información sobre los parámetros acústicos involucrados en las pruebas realizadas, limita la comparación de los resultados obtenidos con niños con visión normal.

Un conjunto de factores relativos a la maduración del sistema auditivo, el crecimiento de la cabeza y del pabellón auditivo, el aprendizaje y la memoria, han sido vinculados a la mejora en el rendimiento en pruebas de localización auditiva que se observa en niños con visión normal durante los primeros años de vida (Hüg \& Arias, 2009). En el caso de los niños ciegos, las demoras en el logro de la conducta de alcanzar un objeto sonoro, han sido asociadas a retrasos en el desarrollo cognitivo y motor, como en la adquisición de la noción de permanencia del objeto (Bigelow, 1986; Fraiberg et al., 1966) y de conductas de locomoción autoiniciadas como arrastrarse, gatear y caminar sin ayuda (Bigelow, 1992; Fraiberg, 1968).

Cabe recordar que, según Piaget (1954), los bebés no son capaces de buscar y obtener un juguete que ha desaparecido de su vista oculto bajo una tela, sino hasta los 8 meses. La adquisición de la noción de permanencia del objeto implica que los niños han adquirido un sistema de representaciones que les permite comprender que un objeto existe de manera independiente de su percepción inmediata y que son capaces de actuar guiados por esta representación. Según esta hipótesis, en ausencia de experiencia visual la adquisición de representaciones sobre el entorno se realiza más lentamente a partir de la información táctil, lo que explicaría la dificultad de esta tarea para los niños ciegos.

Sin embargo, la evidencia obtenida en las últimas décadas ha cuestionado estos postulados. Investigaciones sobre la conducta de alcanzar objetos sin utilizar claves visuales en niños con visión normal, en las que la oscuridad es el "material" con el que se oculta el juguete, han demostrado que no existen diferencias en la edad en la que se observa el primer intento de asir un sonajero en condición de luz y oscuridad (Clifton et al., 1993). Por otra parte, se ha postulado que la tarea de buscar un juguete que se ha ocultado no supone necesariamente la noción de permanencia del objeto (McCall \& Clifton, 1999) y la evidencia obtenida utilizando otros paradigmas experimentales indica que ya a los 3 meses de edad los niños poseen alguna noción de permanencia del objeto (Baillargeon, 1987). Ross y Tobin (1997) señalan al respecto que la ausencia de respuestas de alcance en los niños ciegos no implica necesariamente la existencia de retrasos en su desarrollo cognitivo, sino que puede explicarse por rasgos propios de la actividad exploratoria de estos niños, como por ejemplo, su dificultad para conocer las posibilidades para la acción que ofrece un objeto sonoro nuevo.

$\mathrm{Al}$ respecto, cabe destacar que perspectivas teóricas como la teoría ecológica o de las contingencias sensorio-motoras de la percepción, sostienen que la experiencia perceptual no es el producto de representaciones internas, sino de una manera de "actuar" que involucra también un conocimiento práctico sobre las posibles conductas y las contingencias sensorio-motoras asociadas ( $\mathrm{O}^{\prime}$ Regan \& Noë, 2001), por ejemplo, los cambios en la sensoriopercepción que se producen cuando el niño mueve la cabeza en dirección a una fuente sonora. Desde esta postura, el desarrollo perceptual se produce como resultado de la actividad exploratoria, mediante 
la cual el bebé aprende a realizar diferenciaciones y percibir relaciones multimodales cada vez más finas y complejas. Las dificultades de algunos niños ciegos para lograr conductas como gatear para alcanzar un objeto sonoro pueden deberse a la ausencia de información sensorial complementaria, simultánea, sucesiva y contingente sobre el propio movimiento y el objeto a alcanzar.

Al respecto, Ashmead et al. (1998) postulan que el niño ciego desarrolla las mismas habilidades de audición espacial que el niño con visión normal debido a que, en ausencia de visión, en etapas tempranas el sistema auditivo se calibra a partir de la percepción de los cambios en las claves auditivas que provoca el propio movimiento, como girar la cabeza o caminar. La realización de estudios que apliquen procedimientos psicofísicos para estimar umbrales de localización en los primeros años de vida permitirá delimitar la influencia de factores perceptuales, motores y cognitivos en el rendimiento.

Cabe destacar que las demoras observadas en los niños ciegos durante la primera infancia en tareas de localización auditiva parecen estar en contradicción con los resultados obtenidos por el estudio de Ashmead et al. (1998), descrito anteriormente, realizado con niños ciegos de mayor edad (entre 6 y 20 años, media de 14 años) y otras investigaciones llevadas a cabo con participantes ciegos adultos, que muestran un rendimiento similar e incluso superior, al de las personas con visión normal. Lessard, Paré, Lepore y Lassonde (1998) evaluaron el rendimiento de adultos ciegos congénitos y con visión normal en una tarea de localización de sonidos directos con dos oídos y con un oído, en el plano horizontal. Los resultados mostraron que cuando tenían disponibles claves binaurales, el rendimiento de los participantes ciegos congénitos fue tan preciso como el de las personas con visión normal y, cuando solo disponían de claves monoaurales, algunos participantes ciegos mostraron un rendimiento superior.

Otros estudios realizados con personas adultas en tareas de atención selectiva auditiva y localización de sonidos, evidenciaron que los participantes ciegos tuvieron un rendimiento superior al de participantes con visión normal en las regiones laterales extremas más difíciles (cercanas a \pm 90 ) (Fieger,
Röder, Teder-Sälejärvi, Hillyard \& Neville, 2006; Röder et al., 1999). En relación con la percepción de distancia, un estudio realizado con personas ciegas adultas y con visión normal, concluyó que los primeros tuvieron un rendimiento superior en una tarea de discriminación de la distancia relativa de fuentes sonoras presentadas en el espacio lejano (a 3 metros) (Voss et al., 2004).

El mejor rendimiento en tareas de localización auditiva ha sido interpretado como evidencia de que las personas ciegas, debido a las condiciones que les impone su discapacidad sensorial, realizan un aprendizaje implícito en habilidades auditivas espaciales que les permite optimizar el procesamiento de la información sonora, especialmente en las condiciones más desfavorables, con respecto a las personas con visión normal (Arias, 2009). Durante la infancia, el niño ciego prestaría más atención a las propiedades espaciales de las fuentes sonoras presentes en el entorno, mientras que el niño con visión normal se basaría principalmente en claves espaciales visuales para guiar su conducta. La atención selectiva del niño ciego a la estimulación auditiva presente en el entorno cotidiano facilitará el paulatino aprendizaje implícito de las propiedades relevantes del ambiente sonoro, lo que explicaría el mejor rendimiento que obtienen posteriormente las personas ciegas adultas en pruebas de audición espacial (Emerson \& Ashmead, 2008).

En síntesis, futuras investigaciones deberán evaluar umbrales de localización auditiva de niños ciegos en la temprana infancia en el plano horizontal, vertical y de discriminación de distancia; elaborar modelos explicativos del proceso de calibración del sistema auditivo en ausencia de información visual; analizar la incidencia de los aspectos motores y cognitivos en el desarrollo de la audición espacial y estudiar las posibles causas de las marcadas diferencias individuales observadas en el rendimiento.

Asimismo, cabe resaltar la importancia de identificar cuáles abordajes metodológicos son los más adecuados para evaluar también aspectos cualitativos en el rendimiento de los niños ciegos. En la literatura examinada en el presente trabajo, se describen algunas conductas particulares de estos niños: una menor habituación de las 
respuestas de alerta al sonido; la preferencia por la voz como estímulo sonoro; el girar la cabeza hasta enfrentar el oído hacia la fuente, como si "miraran" con el oído y la preferencia por responder enfrentando la fuente con un mismo oído. Al respecto, Millar (1998) propone el diseño de estudios destinados a participantes ciegos que analicen el proceso, la heurística mediante la cual estos resuelven pruebas perceptuales y cognitivas, y no centrar el análisis solo en la eficiencia como único parámetro. Ulteriores investigaciones sobre el desarrollo de la localización auditiva en niños ciegos deberán determinar en cada caso cuál es la información sensorial disponible y accesible para el niño en una situación determinada y cuál falta o es inadecuada.

\section{Referencias}

American National Standard Institute. (1999). Acoustical terminology. New York: Autor.

Arias, C. (2009). Ecolocación humana y efecto precedente. Tesis doctoral no publicada, Facultad de Psicología, Universidad Nacional de Córdoba, Córdoba, Argentina.

Ashmead, D. H., Wall, R. S., Ebinger, K. A., Eaton, S. B., Snook-Hill, M. M. \& Yang, X. (1998). Spatial hearing in children with visual disabilities. Perception, 27(1), 105-122.

Baillargeon, R. (1987). Object permanence in three-anda-half- and four-and-a-half- month-old infants. Developmental Psychology, 23(5), 655-664.

Bigelow, A. E. (1986). The development of reaching in blind children. British Journal of Developmental Psychology, 4(4), 355-366.

Bigelow, A. E. (1992). Locomotion and search behavior in blind infants. Infant Behavior and Development, 15(2), 179-189.

Blauert, J. (1997). Spatial hearing: The psychophysics of human sound localization. Cambridge: The MIT Press.

Burlingham, D. (1964). Hearing and its role in the development of the blind. Psychoanalytic Study of the Child, 19, 95-112.

Clifton, R. K. (2001). Lessons from infants: 1960-2000. Infancy, 2(3), 285-309.
Clifton, D., Muir, D., Ashmead, D. \& Clarkson, M. (1993). Is visually guided reaching in early infancy a myth? Child Development, 64(4), 1099-1110.

Clifton, R. K., Perris, E. \& Bullinger, A. (1991). Infants' perception of auditory space. Developmental Psychology, 27(2), 187-197.

Emerson, R. W. \& Ashmead, D. (2008). Visual experience and the concept of compensatory spatial hearing abilities. En J. J. Rieser, D. H. Ashmead, F. F. Ebner \& A. L. Corn (Eds.), Blindness and brain plasticity in navigation and object perception (pp. 367-380). New York: Erlbaum.

Fazzi, E., Lanners, J., Ferrarri-Ginevra, O., Achille, C., Luparia, A., Signorini, S. \& Lanzi, G. (2002). Gross motor development and reach on sound as critical tools for the development of the blind child. Brain and Development, 24(5), 269-275.

Fazzi, E., Signorini, S., Bomba, M., Luparia, A., Lanners, J. \& Balottin, U. (2011). Reach on sound: a key to object permanence in visually impaired children. Early Human Development, 87(4), 289-296.

Fieger, A., Röder, B., Teder-Sälejärvi, W., Hillyard, S. A. \& Neville, H. J. (2006). Auditory spatial tuning in late-onset blindness in humans. Journal of Cognitive Neuroscience, 18(2), 149-157.

Field, J., Muir, D., Pilon, R., Sinclair, M. \& Dodwell, P. (1980). Infants' orientation to lateral sounds from birth to three months. Child Development, 51(1), 295-298.

Fraiberg, S. (1968). Parallel and divergent patterns in blind and sighted infants. The Psychoanalytic Study of the Child, 23, 264-300.

Fraiberg, S. \& Freedman, D. A. (1964). Studies in the ego development of the congenitally blind child. The Psychoanalytic Study of the Child, 19, 113-169.

Fraiberg, S., Siegel, B. L. \& Gibson, R. (1966). The role of sound in the search behavior of a blind infant. The Psychoanalytic Study of the Child, 21, 327-357.

Grantham, D. W. (1995). Spatial hearing and related phenomena. En B. C. J. Moore (Ed.), Hearing (pp. 297-345). San Diego, CA: Academic Press.

Hillier, L., Hewitt, K. \& Morrongiello, B. (1992). Infants' perception of illusions in sound localization: Reaching to sounds in the dark. Journal of Experimental Child Psychology, 53(2), 159-179. 
Hüg, M. X. (2011). Aspectos evolutivos de la localización sonora en niños: implicaciones en el desarrollo de la habilidad de ecolocación. Tesis doctoral no publicada, Facultad de Psicología, Universidad Nacional de Córdoba, Córdoba, Argentina.

Hüg, M. X. \& Arias, C. (2006, agosto). Consideraciones sobre audición y desarrollo de habilidades espaciales en infantes discapacitados visuales. Trabajo presentado en las XVIII Jornadas Argentinas de Tiflología, La Rioja, Argentina.

Hüg, M. X. \& Arias, C. (2009). Estudios sobre localización auditiva en etapas tempranas del desarrollo infantil. Revista Latinoamericana de Psicología, 41(2), 225-242.

Humphrey, G. K., Dodwell, P. C., Muir, D. W. \& Humphrey, D. E. (1988). Can blind infants and children use sonar sensory aids? Canadian Journal of Psycho$\log y, 42(2), 94-119$.

Leonhardt, M. (2000). Evaluación del comportamiento en el recién nacido deficiente visual: un estudio de aplicación de la Escala de Brazelton. Integración: Revista sobre Ceguera y Deficiencia Visual, 33, 5-13.

Leonhardt, M. (2001). El déficit visual: la ceguera y baja visión. En I. Bustos Sánchez (Coord.), La percepción auditiva: un enfoque transversal (Vol. 1). Madrid: ICCE.

Lessard, N., Paré, M., Lepore, F. \& Lassonde, M. (1998). Early-blind human subjects localize sound sources better than sighted subjects. Nature, 395(6699), 278-280.

Litovsky, R. Y. (1997). Developmental changes in the precedence effect: Estimates of minimum audible angle. The Journal of the Acoustical Society of America, 102(3), 1739-1745.

McCall, D. \& Clifton, R. (1999). Infants' means-end search for hidden objects in the absence of visual feedback. Infant Behavior Development, 22(2), 179 195.

Mervis, C. A., Boyle, C. A. \& Yeargin-Allsopp, M. (2002). Prevalence and selected characteristics of childhood vision impairment. Developmental Medicine and Child Neurology, 44(8), 538-541.

Millar, S. (1988). Models of sensory deprivation: The nature/nurture dichotomy and spatial representation in the blind. International Journal of Behavioral Development, 11(1), 69-87.
Moore, B. C. J. (1995). Hearing. Handbook of perception and cognition (2nd. ed.). San Diego, CA: Academic Press.

Morrongiello, B. (1987). Infants' localization of sounds in the median sagittal plane: Effects of signal frequency. The Journal of the Acoustical Society of America, 82(3), 900-904.

Morrongiello, B. (1988). Infants' localization of sounds along the horizontal axis: Estimates of MAA. Developmental Psychology, 24(1), 8-13.

Morrongiello, B. \& Rocca, P. T. (1987). Infants' localization of sounds in the median vertical plane: Estimates of minimum audible angle. Child Development, 43(2), 181-193.

Morrongiello, B. \& Rocca, P. T. (1990). Infants' localization of sounds within hemifields: Estimates of minimum audible angle. Child Development, 61(4), 1258-1270.

Muir, D., Clifton, R. K. \& Clarkson, M. (1989). The development of a human auditory localization response: A U-Shaped function. Canadian Journal of Psychology, 43(2), 199-216.

Muir, D. \& Field, J. (1979). Newborn infants orient to sounds. Child Development, 50, 431-436.

Norris, M., Spaulding, P. \& Brodie, F. (1957). Blindness in children. Chicago: University of Chicago Press.

O'Regan, J. K. \& Noë, A. (2001). A sensorimotor account of vision and visual consciousness. Behavioral and Brain Sciences, 24(5), 939-973.

Perrott, D. R., Saberi, K., Brown, K. \& Strybel, T. Z. (1990). Auditory psychomotor coordination and visual search performance. Perception and Psychophysics, 48(3), 214-226.

Piaget, J. (1954). The construction of reality in the child. New York: Basic Books.

Röder, B., Teder-Sälejärvi, W., Sterr, A., Rösler, F., Hillyard, S. A. \& Neville, H. J. (1999). Improved auditory spatial tuning in blind humans. Nature, 400 (6740), 162-166.

Ross, S. \& Tobin, M. J. (1997). Object permanence, reaching, and locomotion in infants who are blind. Journal of Visual Impairment Eु Blindness, 91(1), 25-32.

Schneider, B. A. \& Trehub, S. E. (1992). Sources of developmental change in auditory sensitivity. En L. A. Werner \& E. W. Rubel (Eds.), Developmen- 
tal psychoacoustics (pp. 3-46). Washington, DC: American Psychological Association.

Voss, P., Lassonde, M., Gougoux, F., Fortin, M., Guillemot, J. P. \& Lepore, F. (2004). Early- and late-onset blind individuals show supra-normal auditory abilities in far-space. Current Biology, 14(19), 1734-1738.

Warren, D. H. (1984). Blindness and early childhood development (2nd. ed.). New York: AFB Press. 